\title{
A GEMM interface and implementation on NVIDIA GPUs for multiple small matrices
}

\author{
Chetan Jhurani*, Paul Mullowney \\ Tech-X Corporation \\ 5621 Arapahoe Ave \\ Boulder, Colorado 80303, U.S.A.
}

\begin{abstract}
We present an interface and an implementation of the General Matrix Multiply (GEMM) routine for multiple small matrices processed simultaneously on NVIDIA graphics processing units (GPUs). We focus on matrix sizes under 16 . The implementation can be easily extended to larger sizes. For single precision matrices, our implementation is $30 \%$ to $600 \%$ faster than the batched cuBLAS implementation distributed in the CUDA Toolkit 5.0 on NVIDIA Tesla K20c. For example, we obtain 104 GFlop/s and 216 GFlop/s when multiplying 100,000 independent matrix pairs of size 10 and 16, respectively. Similar improvement in performance is obtained for other sizes, in single and double precision for real and complex types, and when the number of matrices is smaller. Apart from our implementation, our different function interface also plays an important role in the improved performance. Applications of this software include Finite Element computation on GPUs.
\end{abstract}

Keywords:

NVIDIA CUDA, GPU, GEMM, BLAS, cuBLAS, Parallel programming, Dense linear algebra

\footnotetext{
* Corresponding Author

Email addresses: chetan.jhurani@gmail.com (Chetan Jhurani), paulm@txcorp.com (Paul Mullowney)
} 


\section{Introduction}

Implementations of the General Matrix Multiply (GEMM) routine typically achieve a large fraction of peak speed on modern multi-core hardware. However, because of hardware characteristics, near peak performance is achieved for large matrix sizes, which is usually in hundreds or even thousands $[1,2]$, or for sizes that are integer multiples of hardware-specific dimensions.

Although such large dense matrices are important in many applications and also help in showcasing the impressive speeds, many other applications require multiplication of multiple independent small matrices, each having identical size. Such a situation is common in finite element methods for example $[3,4,5,6,7,8]$. Even if sizes are different, one can partition matrices so that sets with identical matrix sizes are obtained.

Our goal is to obtain high performance when processing multiple small matrices, numbering in thousands or more, on NVIDIA GPUs. The definition of small is not standard and cannot be invariant with respect to hardware trends. Still, 'small' can be taken to be a size for which high performance is difficult to achieve in a parallel environment unless multiple independent matrices are involved. Note that even for large matrices, efficient implementations of GEMM multiply sub-blocks which are smaller and achieve high performance. However, the difference here is that our small matrices are independent and are of different sizes.

Achieving high performance GEMM for small matrix sizes, when compared to large sizes, is inherently difficult because each entry is used fewer times after it is copied from main memory to registers. However, developing a high-quality GEMM implementation is crucial. Apart from its inherent utility, fast GEMM is also a basis for the speeds achievable in other level-3 BLAS functions [9].

In the context of GPUs, the need for a capability to multiply pairs of small matrices was recognized by NVIDIA. An implementation focused on small matrix sizes was released with the Compute Unified Device Architecture (CUDA) toolkit version 4.1 in the cuBLAS library. The so-called "batched" implementation (function name cublasXgemmBatched, $\mathrm{X}=\mathrm{S} / \mathrm{D} / \mathrm{C} / \mathrm{Z}$ for different data types) is significantly faster than two other possibilities - one using CUDA streams and the original non-streamed version intended for large matrices. This improvement in speed due to batching holds for matrix sizes up to roughly 100. However, except for small matrices with sizes that are 
integral multiples of 16 , the achieved performance is still a small fraction of peak GPU speed [10]. One can also observe that the function's performance is quite sensitive to the matrix size being a multiple of 16 . For example, its speed in multiplying 100,000 single precision matrix pairs of size 16 is 134 GFlop/s. But it drops to 105 GFlops/ for size 15, and for size 17 it drops even more to 32 GFlop/s. These numbers are using the "batchCUBLAS" example program run on Tesla K20c, a device for which the peak single precision speed is $3.52 \mathrm{TFlop} / \mathrm{s}$.

Another issue is that the function interface of cublasXgemmBatched is designed around pointers to the matrix pointers, which increases its applicability when calling from languages that can deal with pointers to pointers and if different matrices were allocated separately. But this feature also makes it less efficient because one has to transfer each pointer as extra data.

Our objectives in this contribution are to achieve higher performance GEMM for small matrices on GPUs and also to provide an alternative interface that uses a second-level leading dimension (a BLAS/LAPACK terminology [11]). Using our interface and the implementation, we achieve an improvement in speed that varies in $[30 \%, 600 \%]$ compared to the reference cuBLAS batched implementation for single precision matrices. The improvement is in the higher range when the matrix sizes are smaller. Similar improvements are observed for other scalar types.

If we just use the cuBLAS-like interface but the underlying implementation is ours, the speedup is lower but still significant. In this case, compared to the reference, we achieve a more modest speed improvement (or a minor reduction in a tiny fraction of cases) in $[-30 \%, 300 \%]$. We also include discussion of a few $\mathrm{C}++$ template related features that help in achieving generality and high performance.

Our focus here is on square matrices of size 16 or less but the method can be easily implemented for rectangular cases and for larger sizes. The main reason we have focused our attention on matrices of size 16 and less is that such sizes correspond to the polynomial degrees that are traditionally used in high-order finite elements $[3,4,5,6,7,8]$.

Here is an outline of the paper. In Section 2 we briefly describe our notation for batched GEMM. Sections 3 and 4 are devoted to describing the cuBLAS interface that we use as reference and the design trade-offs associated with it. In Sections 5 and 6 we describe the interface of our CUDA kernel and the function wrapping it. There we also describe various optimizations possible due to $\mathrm{C}++$ features. We show our implementation in Section 7 . 
We discuss the performance of our implementation from various viewpoints in Section 8.

\section{Multiple GEMMs}

Let op (standing for operation) refer to a mapping from matrices to matrices, such that $o p(A)=A$ or $A^{T}$ or $A^{*}$ depending on an extra variable that can contain three values. The superscripts $T$ and $*$ stand for transpose and Hermitian-transpose, respectively. Consider real or complex matrices $A^{p}, B^{p}, C^{p}$ for $p=1,2, \ldots, N$. Here $N$ stands for the batch size. The matrices can be stored in single precision or double precision. Using the BLAS notation, $C$ is $m \times n, o p(A)$ is $m \times k$, and $o p(B)$ is $k \times n$.

Let $\alpha$ and $\beta$ be given scalars. Our goal is to compute

$$
C^{p} \leftarrow \alpha o p\left(A^{p}\right) o p\left(B^{p}\right)+\beta C^{p}
$$

for $p=1,2, \ldots, N$ independently in the CUDA environment. Note that in this context the operation op acting on $A$ can be different than the one acting on $B$, which leads to 9 combinations in all.

\section{NVIDIA cuBLAS interface}

We first provide a few details of our baseline. The batched implementation in NVIDIA cuBLAS versions 4.1,4.2, and 5.0 for computing the output in Equation (1) is a $\mathrm{C}$ function with a signature given below. The letter $T$ (standing for type) refers to the concrete type, which can be S,D, C, Z for float, double, cuComplex, or cuDoubleComplex respectively. The detailed documentation is available online [10]. The interface is easy to understand and almost natural for a BLAS/LAPACK user. The important part of the interface is that arrays of matrices as passed as follows. Only "A" is shown below.

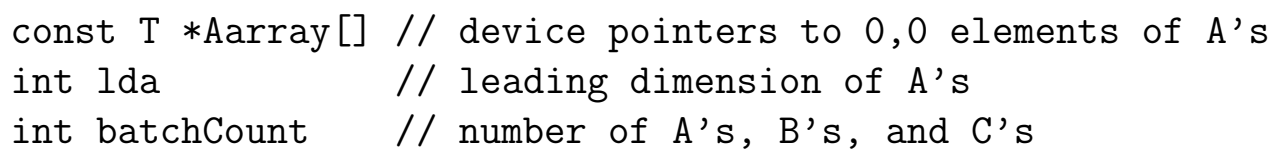

Thus, Aarray [k] is the pointer to the $k^{\text {th }}$ matrix and Aarray [k] [i+lda*j] is its $(i, j)$ entry. 


\section{Design trade-offs in the cuBLAS interface}

The cuBLAS interface is natural when using multiple matrices that may have been created and stored independently or if the number of matrices is dynamically changing. However, the interface has a few design issues. One would have to collect the pointers to the matrices and copy the three arrays (for $A, B, C$ ) to the device. This leads to additional overhead due to (possibly) multiple allocations, transmission of pointers, as well as memory access during kernel execution.

An alternative way to use the cuBLAS interface is to allocate a large chunk of memory once and store pointers to appropriate positions so that it looks like a 3-D array - a uniformly-offset collection of matrices of identical size. However, even now, neither the function invocation nor the kernel execution have used the knowledge that the matrices are uniformly-offset. This leads us to the hypothesis that a somewhat less general but a more efficient as well as appropriate way is to not pass pointers to pointers, but use a second leading dimension to indicate the offset between adjacent matrices. One needs to pass pointer to the 0,0 element of only the first matrix and just a single extra integer for another leading dimension.

To test this hypothesis, we will show results from two versions of our kernel, one with the interface like the one in cuBLAS and other with the 3-D array interface. In both cases, the implementation is identical as far as logically possible. It will be shown that the second one increases performance even after transmission overhead associated with creating and passing pointers to pointers is ignored (see Section 8). Hence, we can conclude that in the regime of large batch size and small matrix sizes (less than or equal to 16), the natural interface is the one that uses a 3-D array to represent a collection of 2-D matrices.

\section{A specialized function interface}

As mentioned earlier, we develop kernels with two kinds of interfaces to test which interface gives us the better performance. The first one resembles the cuBLAS interface. We call it TGEMM_multi_nounif, where 'multi' stands for multiple and 'nounif' is for 'not uniform'. The second one is called TGEMM_multi_uniform and is the focus of this research. It uses a secondlevel leading dimension. Hence we show the second and new interface only. We only show the changes made to storage of matrices. Only "A" is shown below. 


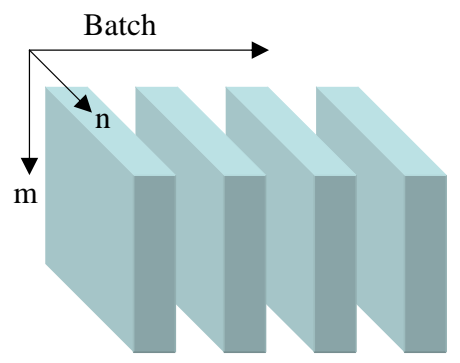

Figure 1: A layout of matrices in the TGEMM_multi_uniform kernel. The dimensions $m$ and $n$ refer to the output matrices $C^{p}$. We have $l d c \geq m$ and $l d c 2 \geq l d c * n$.

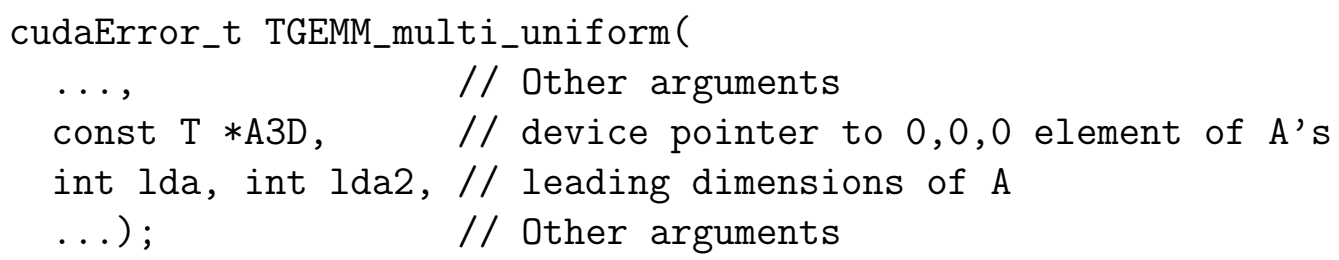

The second leading dimension (lda2) is the offset between adjacent 2D matrices. The $(i, j)$ entry in $k^{t h} A$ matrix in the batch is A $[i+1 d a * j+1 d a 2 * k]$. Similar relations hold for $B$ and $C$. Figure 1 shows the layout of the batch of $C$ matrices.

\section{A $\mathrm{C}++$ kernel interface}

We now present the $\mathrm{C}++$ interface for the CUDA kernel underlying the TGEMM multi_uniform function. It is intended for multiplication of square matrices.

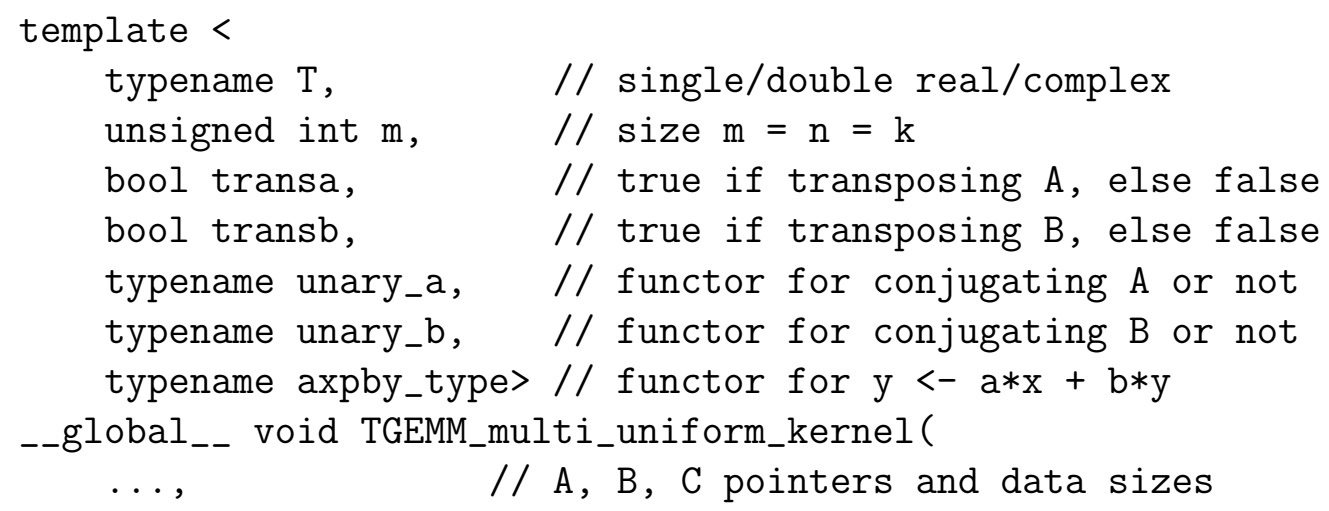




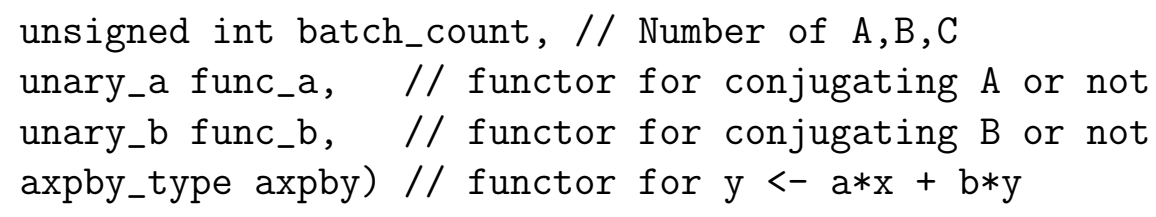

$\mathrm{A} \mathrm{C}++$ functor (or function object) is useful for compile-time polymorphism [12] and is important for achieving high-performance. The essential idea is to move Boolean checks from the innermost loop to outside so it will lead to fewer branching possibilities. The drawback is that it leads to larger executable code size when a large number of independent template permutations are instantiated. Note that our intent is not at all to suggest that cuBLAS or other libraries do not internally use functors or templates like these.

We briefly discuss the specific rationale and a few candidates for the axpby_type and unary_a argument types. These functors are applied entrywise to pairs of scalars. In many cases, the GEMM parameters $\alpha$ (for $a$ ) and $\beta$ (for $b$ ) are not arbitrary scalars but are simple values like $-1,0$ or 1 . In such cases explicit multiplication is not needed. For example, one may just want to compute $C \leftarrow A B$ (so $\alpha=1, \beta=0)$. The effect on performance can be noticeably large when $\beta$ is zero but a general code still reads the output entry from global memory, multiplies it by $\beta$, and then writes the final sum back to global memory. Such extra computation and memory movement can be prevented by passing a stateless object of the following type.

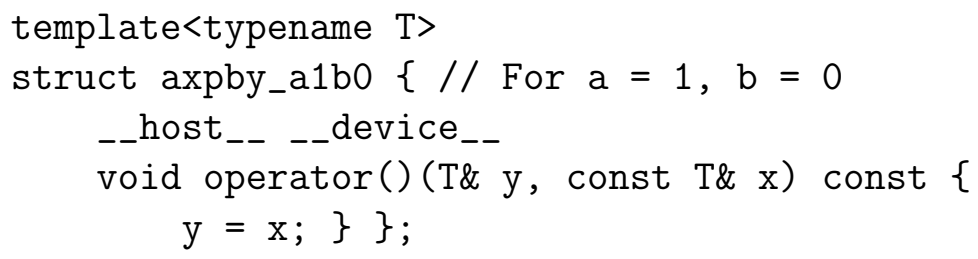

The following functor can be used for general $a$ and $b$.

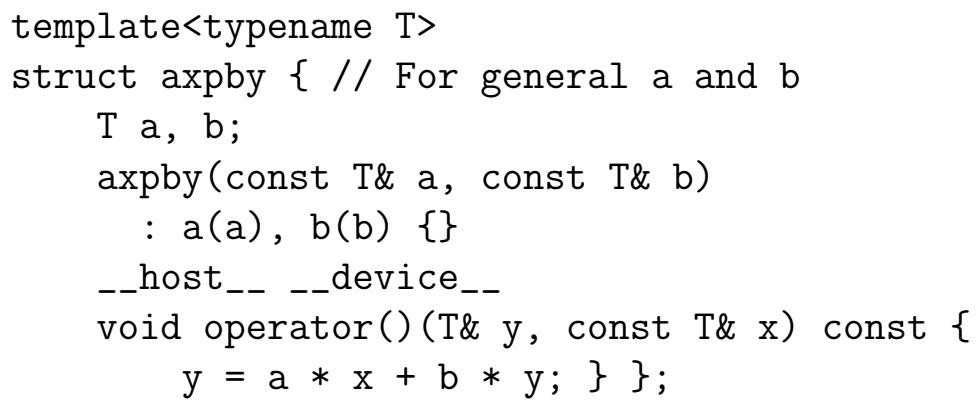


One would then call axpby(C_entry, (AB)_entry) in the CUDA kernel for each entry. Functors for other special combinations of $a$ and $b$ can also be used. Compiler optimizations inline all these calls.

In a similar vein, when one wants to apply conjugates for complex scalars, functors can be used that return the conjugate of a given entry. Here is an example for double precision complex.

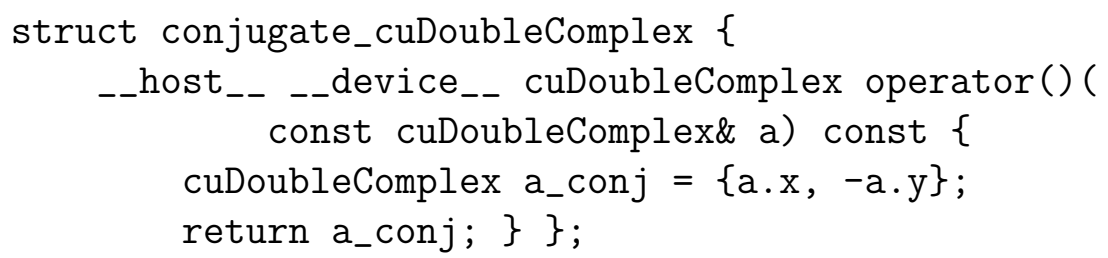

\section{CUDA kernel implementation}

Here we show our implementation of TGEMM_multi_uniform_kernel. In reality, we have two distinct kernels. We found experimentally that the second kernel was faster than the first one for sizes 15 and 16 and we used that for the results. For other (smaller) sizes, the first one was faster.

Our first kernel works for each square matrix size in 1-16. All the threads collectively read $A$ and $B$ matrices to shared memory. Each thread within a thread-block computes one entry of each output matrix $C$ in the batch. Thus, for processing $m \times m$ matrices we launch $m^{2}$ threads. Here is the code for this kernel. The function signature and template types are shown in 6 , so we do not repeat them here.

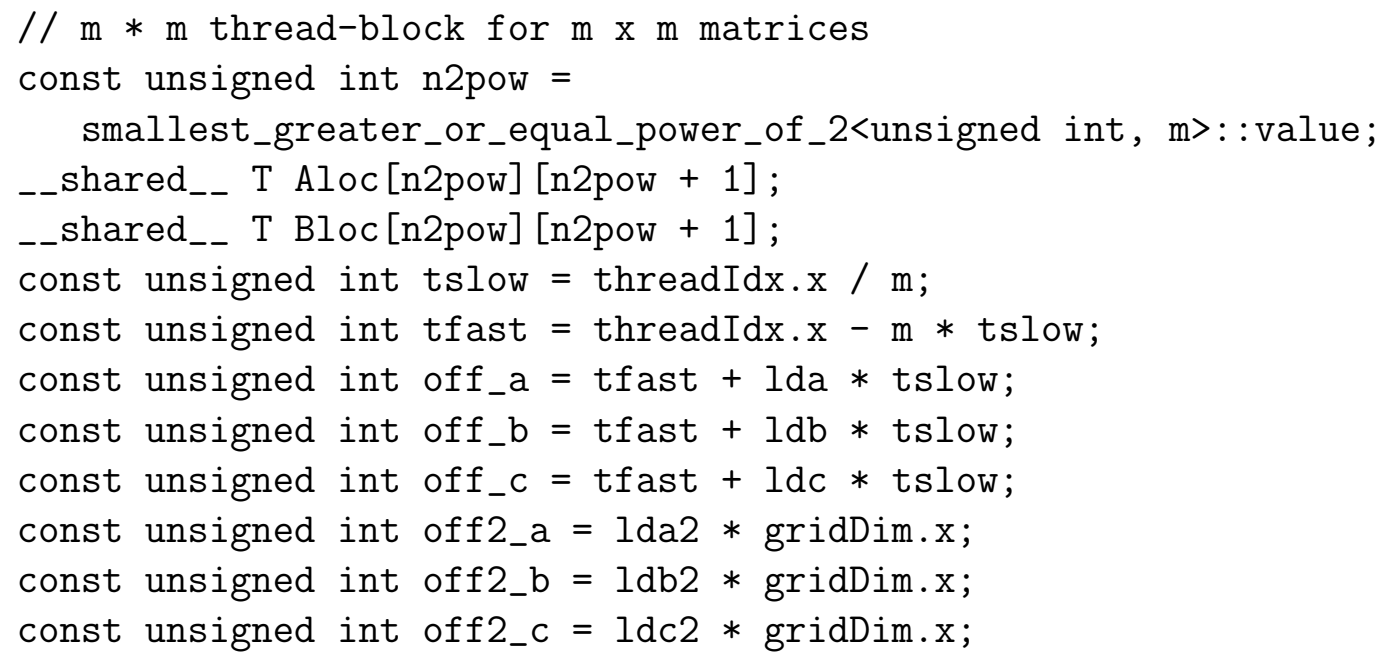




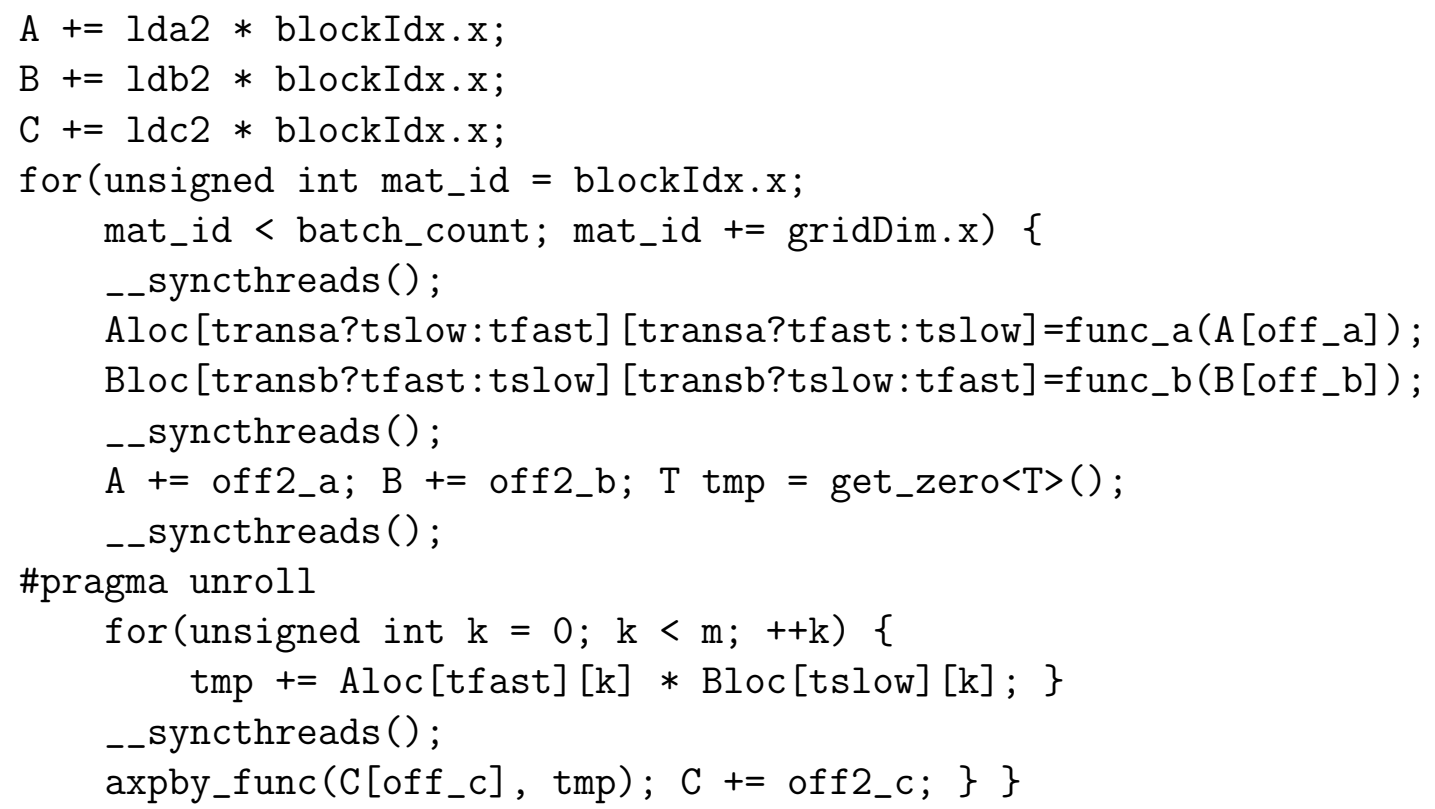

The second kernel can be used for square matrices where the matrix size can be factorized into a product of two numbers. For example, $m=15$ can be factorized as $3 \times 5$ or $5 \times 3$. Say $m=m_{1} m_{2}$. The order is important. We launch a 2-D CUDA block of size $m$ times $m_{2}$. Each thread reads multiple columns of $A$ and $B$ into the shared memory and computes $m_{1}$ columns of $C$. This kernel is inspired from the one implemented in MAGMA [1]. Since similar code exists in MAGMA and we modified it solely for special matrix sizes (15 and 16), we do not show its implementation.

For matrices that are not square, one can use these square matrix kernels to multiply sub-blocks. One can design more specialized kernels of a given matrix size so that the performance can be improved further. However, we have not pursued this task.

\section{Performance results}

Our test hardware is a Tesla K20c GPU, which has a peak performance of $3.52 \mathrm{TFlop} / \mathrm{s}$ and $1.17 \mathrm{GFlop} / \mathrm{s}$ in single and double precision, respectively. We work with the ECC (error-correcting code) mode turned off for all cases. Our code has been compiled with - arch=sm_35 -03 options using the CUDA 5.0 toolkit.

Each experiment is designed to understand the performance variation when a set of parameters is changed. Thus, it is important to remember the 
basic set of parameters around which the variations are tested. This is our basic set of parameters.

- We use 100,000 as batch size.

- We use 2000 thread-blocks.

- We use our kernel that takes second-order leading dimension parameter.

- The no transpose or conjugate-transpose operation is our baseline.

- The memory layout of matrices is such that all the leading dimension parameters are the smallest they can be for the given matrix size.

We clearly mention when some of these are changed in an experiment. The choices above have been made just to design concrete experiments. They are not a restriction in any way.

In our results, the suffix 'unif' is used to specify the leading dimension based kernel, the suffix 'nounif' is used for our implementation with cuBLAS like interface, and the suffix 'cuBLAS' is used for the cuBLAS implementation.

We have used the convention that $2 \mathrm{~m}^{3}$ flops are required to perform GEMM on two real $m \times m$ matrices, for all $\alpha$ and $\beta$ and whether transpose or conjugate-transpose is chosen or not. For complex matrices, we use $8 \mathrm{~m}^{3}$. The "3M" method [13] is not used for complex matrix multiplication. For timing purposes, each kernel run was performed 5 times and we took the median of the time reported by CUDA timers after the device was synchronized.

\subsection{Block size and batch size}

Our first goal is to determine a CUDA kernel block size for large number of matrices such that the performance is close to maximum. We do this for general values of $\alpha$ and $\beta$.

Figure 2 shows the speed achieved when multiplying $N=100,000$ independent single-precision square matrix pairs of different sizes. Clearly, there is very little to gain when running a kernel with more than 2000 blocks. We chose $N$ matrices in a batch to stay well within the limits of GPU memory. For example, in the largest case of double precision complex matrices, one would consume roughly $1.23 \mathrm{~GB}$ in storing $100,000 A, B$, and $C$ matrices. Another reason for choosing that number is shown in Figure 3. There is some speed gain when using large batch sizes but as one can extrapolate, the 


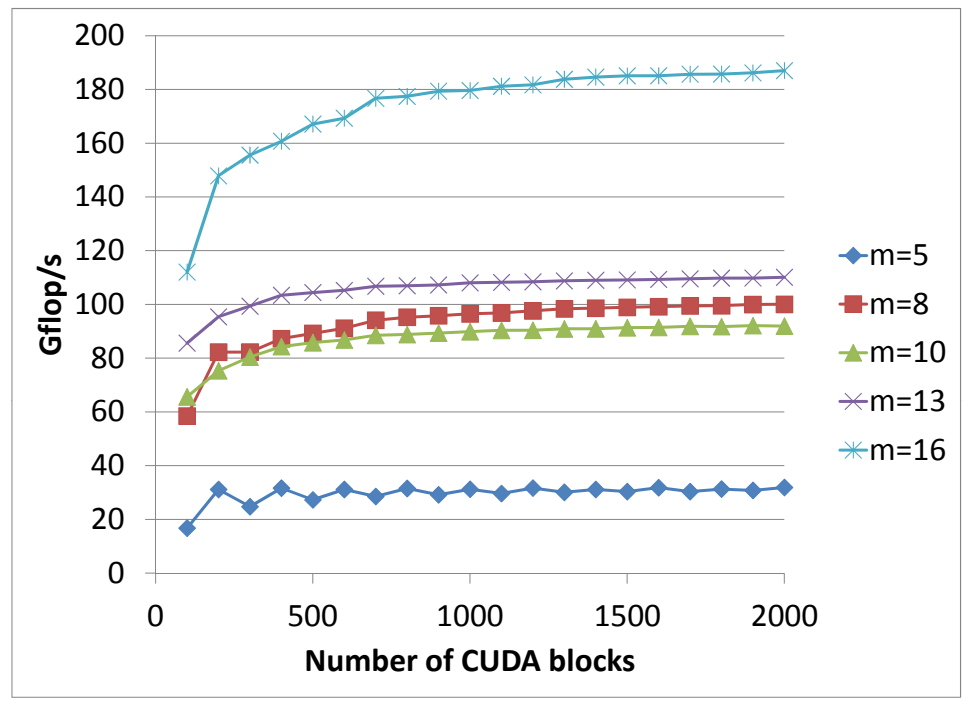

Figure 2: An experiment to determine the effect of CUDA block size. We multiply 100,000 independent single-precision square matrix pairs of different sizes $(m)$.

gains would be minor beyond the threshold of $N=100,000$. We fixed the block size and batch size based on these two experiments. Another result from the second experiment is that even for small batch sizes, say 2000, the performance is not significantly worse compared to large batch sizes.

\subsection{Effect of conjugation or transposition on performance}

In all our other experiments we have not conjugated and/or transposed the matrices that are inputs to GEMM. Here we do. Figure 4 shows that these operations do make our kernels slower, which is expected, but the reduction in speed is minor. The results are shown for single precision complex matrices of size up to 16. This experiment was run for general (randomized) values of $\alpha$ and $\beta$.

\subsection{Improvement due to axpby_type template parameter}

As mentioned earlier, the axpby_type template parameter can be used to reduce computation time for special values of $\alpha$ and $\beta$ while maintaining a general interface. Figure 5 shows the speed and performance gain for single precision matrices of sizes 1-16 when $\alpha=1$ and $\beta=0$. Depending on the matrix size, the gains are between $10 \%$ and $50 \%$ over the code that works for arbitrary $\alpha$ and $\beta$. Hence, the added complexity due to this extra template parameter is worth it. 


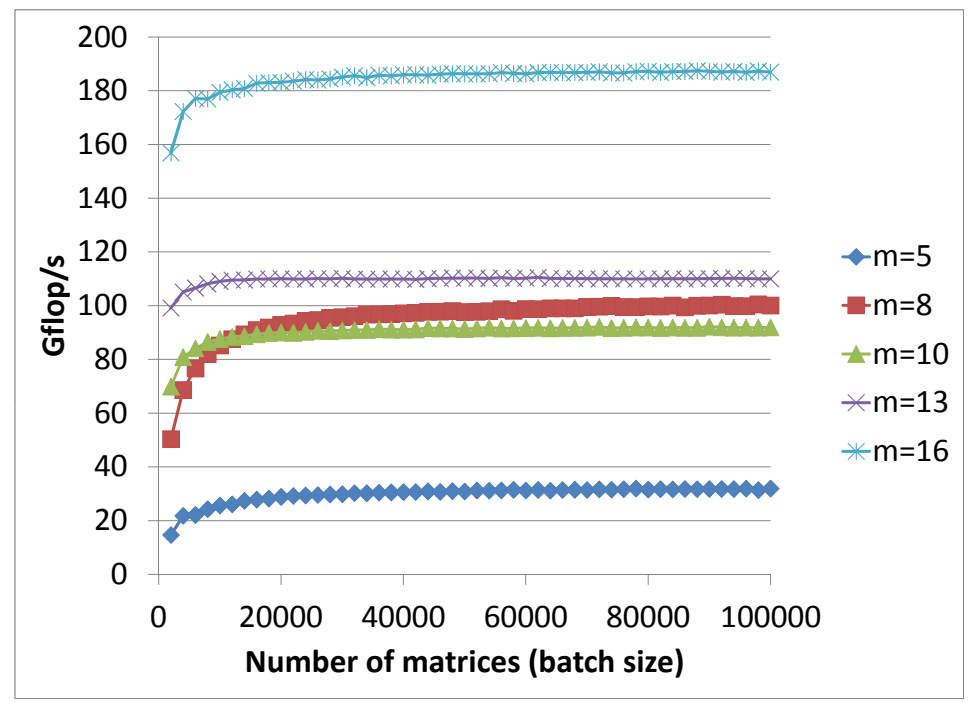

Figure 3: An experiment to show that the performance improvement increases very slowly with increasing batch size for various matrix sizes $m$. 100,000 matrix pairs in a batch is a reasonable parameter to run other experiments.

\begin{tabular}{|c|c|c|c|c|c|c|c|c|c|}
\hline Size & $\mathrm{nn}$ & $\mathrm{nt}$ & nc & tn & tt & tc & $\mathrm{cn}$ & $\mathrm{ct}$ & $\mathrm{CC}$ \\
\hline 1 & 1.000 & 0.777 & 0.769 & 1.014 & 0.777 & 0.770 & 0.999 & 1.011 & 0.996 \\
\hline 2 & 1.000 & 1.277 & 1.003 & 1.000 & 1.273 & 1.001 & 1.277 & 1.270 & 1.277 \\
\hline 3 & 1.000 & 0.787 & 0.783 & 0.999 & 0.786 & 0.784 & 0.985 & 0.985 & 0.766 \\
\hline 4 & 1.000 & 0.998 & 0.809 & 0.999 & 0.998 & 0.809 & 0.995 & 0.999 & 0.977 \\
\hline 5 & 1.000 & 0.998 & 0.824 & 0.998 & 0.815 & 0.809 & 0.992 & 0.834 & 0.988 \\
\hline 6 & 1.000 & 1.005 & 0.851 & 1.003 & 0.856 & 0.855 & 1.014 & 0.875 & 0.992 \\
\hline 7 & 1.000 & 1.001 & 0.909 & 1.005 & 0.917 & 0.922 & 1.006 & 0.934 & 1.000 \\
\hline 8 & 1.000 & 1.099 & 0.989 & 1.007 & 1.103 & 0.991 & 1.111 & 1.107 & 1.092 \\
\hline 9 & 1.000 & 0.999 & 0.882 & 1.002 & 0.891 & 0.892 & 1.015 & 0.908 & 0.993 \\
\hline 10 & 1.000 & 1.001 & 0.912 & 1.007 & 0.926 & 0.927 & 1.032 & 0.940 & 1.015 \\
\hline 11 & 1.000 & 1.006 & 0.925 & 1.004 & 0.935 & 0.938 & 1.025 & 0.948 & 1.009 \\
\hline 12 & 1.000 & 1.001 & 0.915 & 0.999 & 0.917 & 0.922 & 1.017 & 0.932 & 0.998 \\
\hline 13 & 1.000 & 1.009 & 0.945 & 0.999 & 0.954 & 0.959 & 1.037 & 0.965 & 1.009 \\
\hline 14 & 1.000 & 1.092 & 0.981 & 0.998 & 1.084 & 0.981 & 1.102 & 1.084 & 1.094 \\
\hline 15 & 1.000 & 0.992 & 1.013 & 0.997 & 1.016 & 0.998 & 0.970 & 1.002 & 0.981 \\
\hline 16 & 1.000 & 0.994 & 0.998 & 1.002 & 1.003 & 1.000 & 0.925 & 0.929 & 0.985 \\
\hline
\end{tabular}

Figure 4: Performance effect of conjugating (c) and/or transposing (t) one or both sets of input single precision complex matrices with the baseline being no operation (n). Slower performance is indicated by darker colors and lower values (less than 1). 


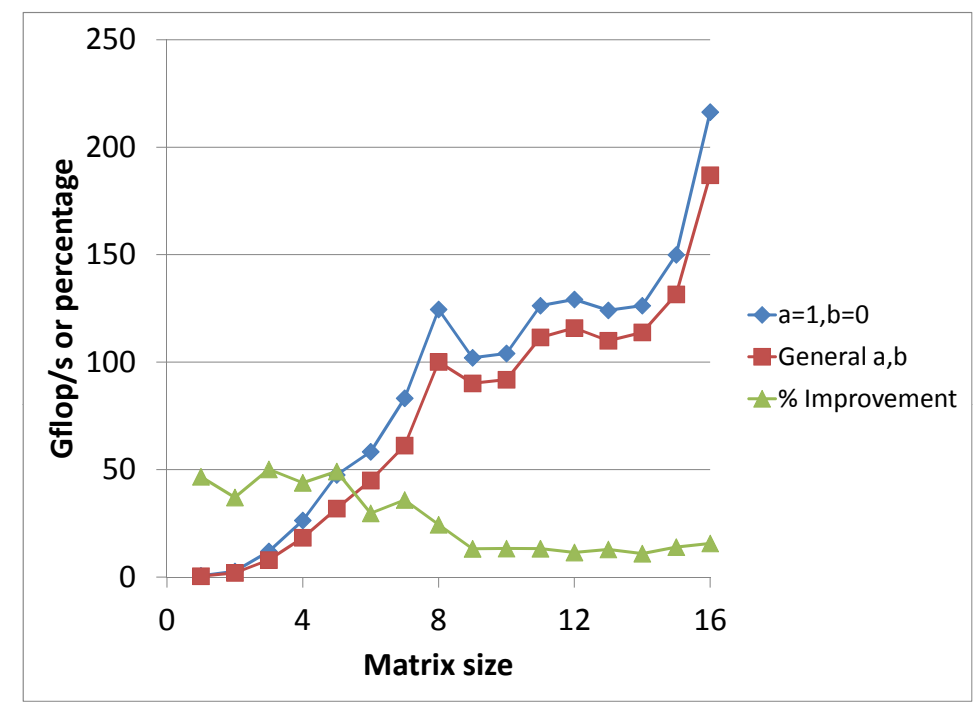

Figure 5: Speed and performance gain for single precision matrices of various sizes when $\alpha=1$ and $\beta=0$ and for general values of these parameters.

\subsection{Speed for various data types and matrix sizes}

We now show the speeds achievable for four data types - real and complex types in single and double precision - for matrix sizes 1-16. We show results where $\alpha=1$ and $\beta=0$ in Figure 6. Naturally, using arbitrary $\alpha$ and $\beta$ will give a slightly lower performance than what is shown. For matrices of size 16 and $\alpha=1$ and $\beta=0$ on NVIDIA Tesla K20c, we reach these GFlop/s rates - 216 for single real, 173 for double real, 609 for single complex, and 217 for double complex. For general $\alpha$ and $\beta$, the speeds are lower by roughly 15\%-30\% for matrices of size 16 .

\subsection{Performance comparison with cuBLAS}

We now compare cuBLAS and our two kernels - one which uses the second-order leading dimension and one using pointers to pointers just like cuBLAS batched interface.

As mentioned earlier, we have ignored memory allocation, deallocation, and transmission overhead required in cuBLAS for working with pointers to pointers. We have measured only the time spent in calling GEMM. Similarly, we have ignored timing these steps for out kernels, but naturally this time will be less than what is required in cuBLAS interface. 


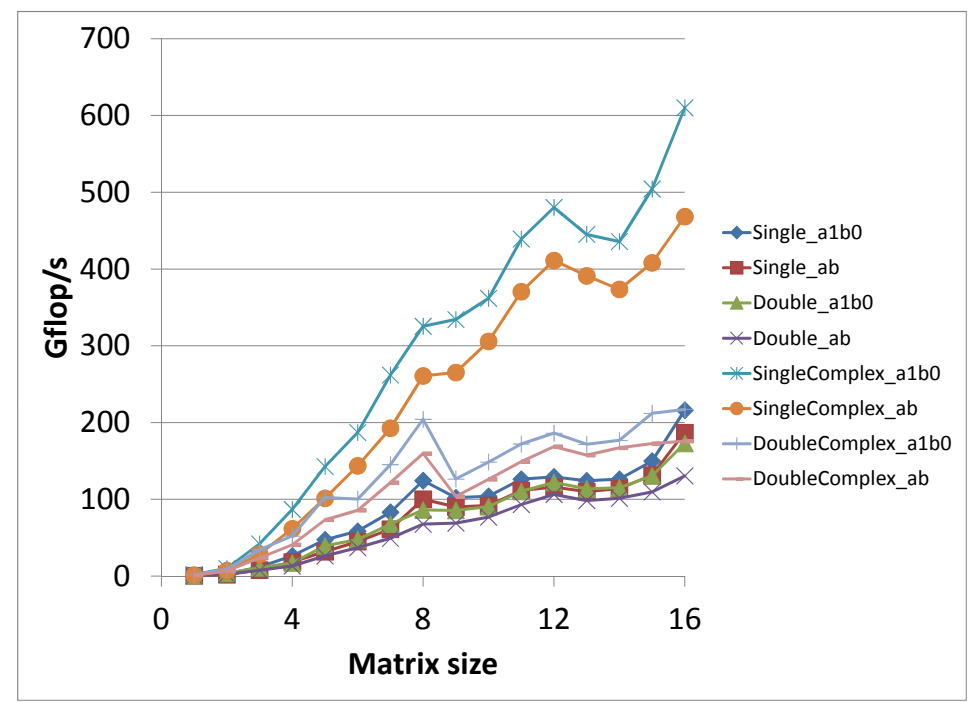

Figure 6: Batched GEMM speed on NVIDIA Tesla K20c for various matrix sizes, various data types, and when $\alpha=1$ and $\beta=0$ (a1b0) or they are general values (ab).

See Figure 7 for the real case and Figure 8 for the complex case. Both our kernels are noticeably faster than cuBLAS for matrix sizes under 16 except for a handful of cases. The second order leading dimension based kernel, which is the one we intend to use, is slower in just one case out of all by $5 \%$.

The results show that part of the improvement is due to our implementation and part of it is due to the changes in interface. Figure 9 shows the relative improvement for various matrix sizes in the case of improved interface. It can be up to $600 \%$ for small sizes. Table 1 lists the speed we achieve when multiplying 100,000 independent matrix pairs of various sizes using the TGEMM multi_uniform kernel on NVIDIA Tesla K20c.

\section{Discussion}

We have described a new interface and two improved implementations of a batched GEMM routine. Another interesting batched GEMM case is when one matrix, either the one on the left or right, is fixed and one has to multiply it with a batch of other matrices. This can be treated by a nonbatched GEMM implementation by concatenating matrices but high performance may not be readily possible because the non-batched GEMM might not be designed for such cases. However, based on our experience in this 


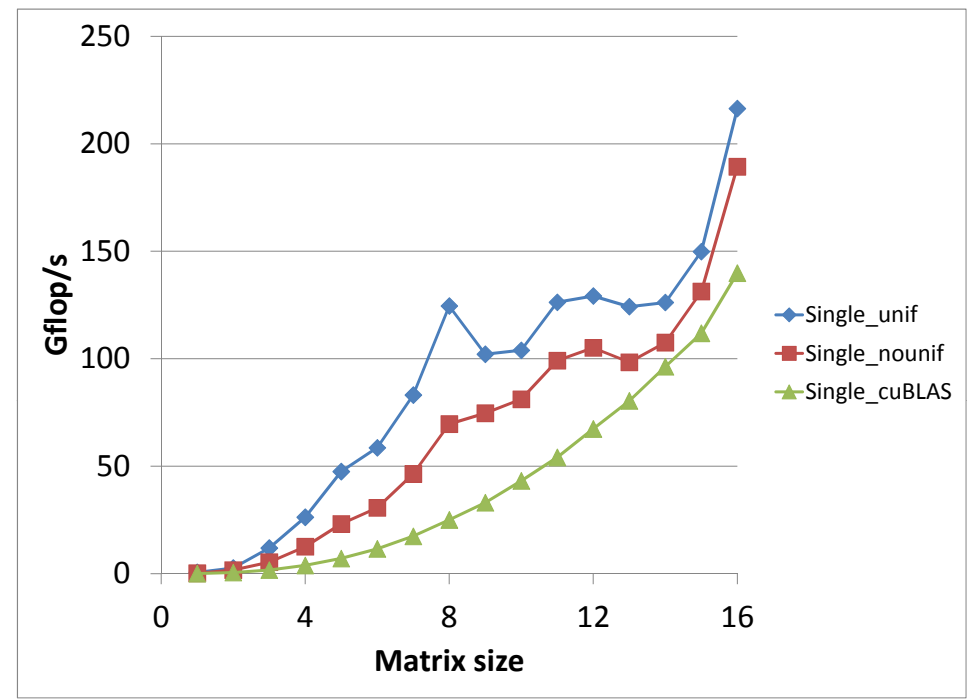

(a) Single precision real

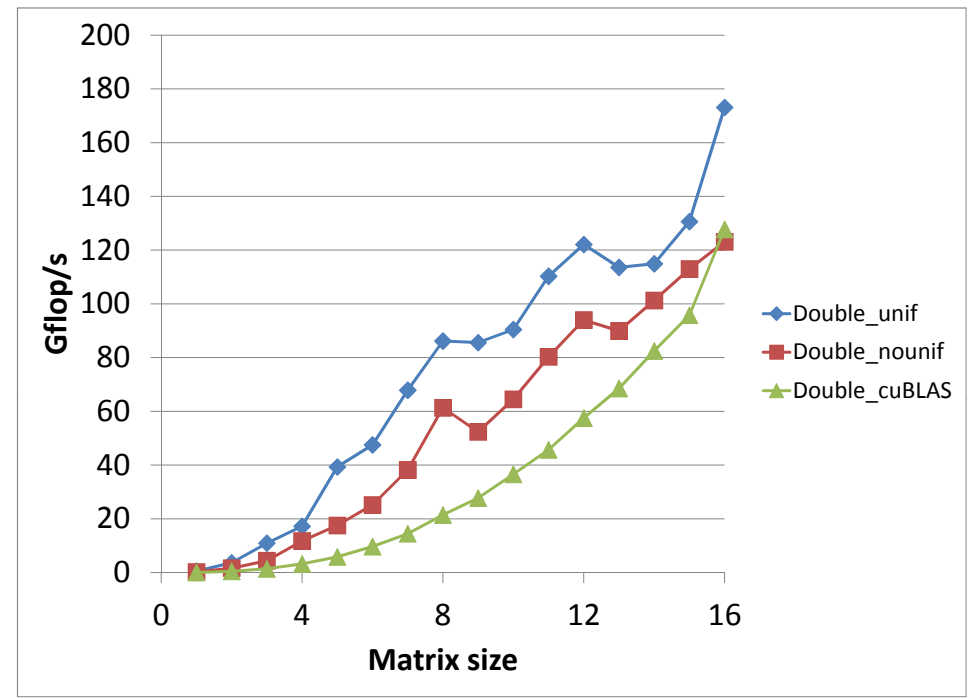

(b) Double precision real

Figure 7: Comparison of the speed of our two kernels and cuBLAS batched implementation for real scalars and $\alpha=1$ and $\beta=0$. Relative improvement is shown in Figure 9. 


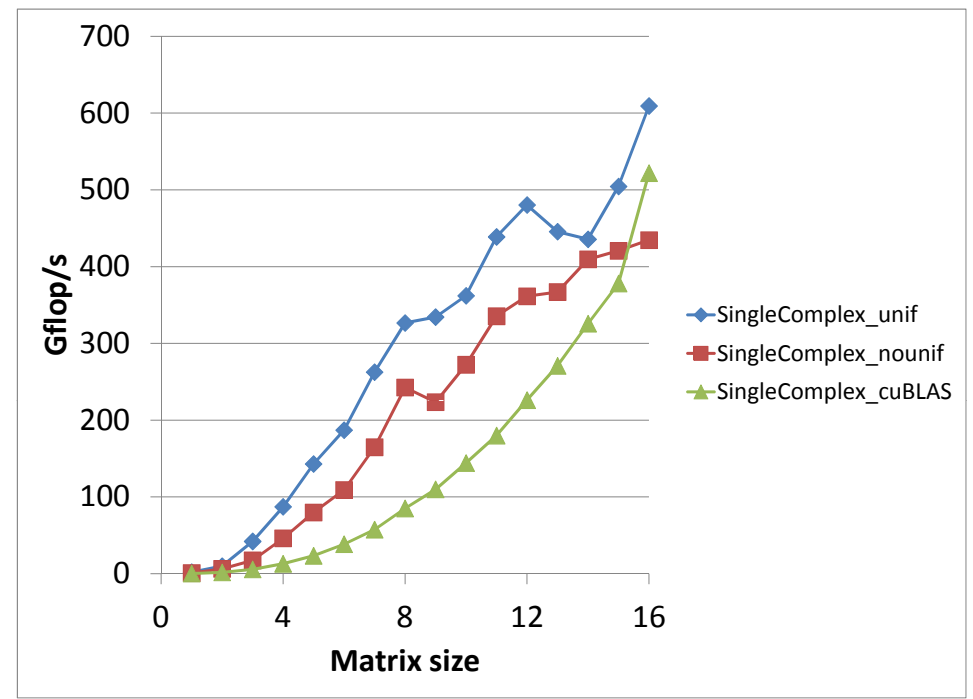

(a) Single precision complex

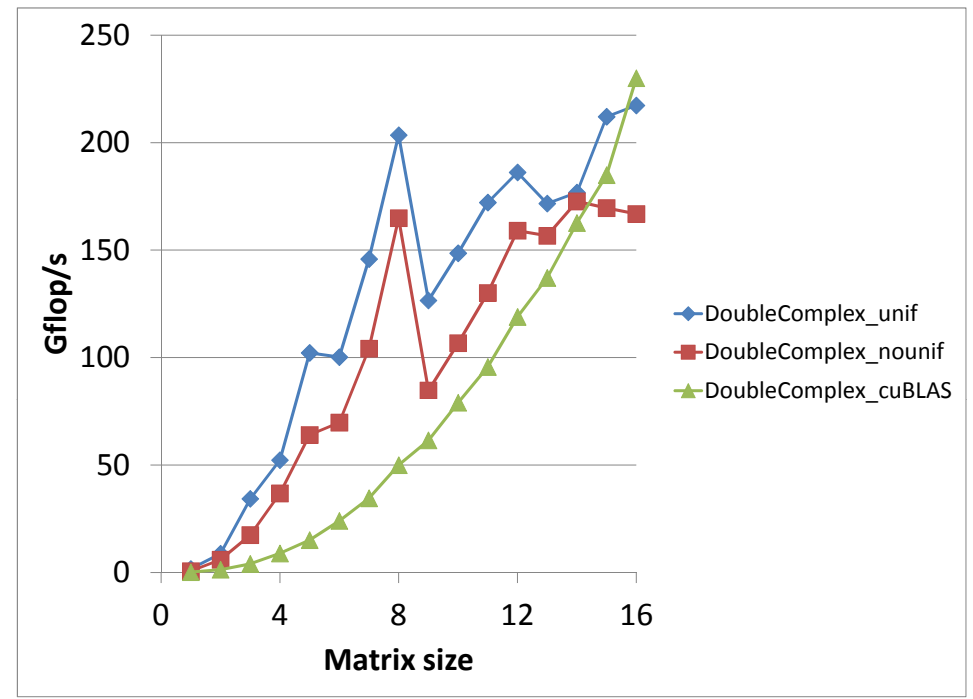

(b) Double precision complex

Figure 8: Comparison of the speed of our two kernels and cuBLAS batched implementation for complex scalars and $\alpha=1$ and $\beta=0$. Relative improvement is shown in Figure 9 . 


\begin{tabular}{rrrrr}
\hline Size & Single-R & Double-R & Single-C & Double-C \\
\hline \hline 1 & 1 & 1 & 2 & 2 \\
2 & 3 & 4 & 10 & 9 \\
3 & 12 & 11 & 42 & 34 \\
4 & 26 & 17 & 87 & 52 \\
5 & 48 & 39 & 143 & 102 \\
6 & 59 & 48 & 187 & 100 \\
7 & 83 & 68 & 263 & 146 \\
8 & 125 & 86 & 327 & 204 \\
9 & 102 & 86 & 334 & 127 \\
10 & 104 & 90 & 362 & 149 \\
11 & 126 & 110 & 439 & 172 \\
12 & 129 & 122 & 480 & 186 \\
13 & 124 & 114 & 446 & 172 \\
14 & 126 & 115 & 436 & 177 \\
15 & 150 & 131 & 504 & 212 \\
16 & 216 & 173 & 609 & 217 \\
\hline
\end{tabular}

Table 1: The GFlop/s rates when multiplying 100,000 independent matrix pairs of various sizes using the TGEMM_multi_uniform kernel on NVIDIA Tesla K20c. The comparison with corresponding numbers in cuBLAS are made in Figure 7 and Figure 8 . The suffixes $\mathrm{R}$ and $\mathrm{C}$ stand for real and complex, respectively.

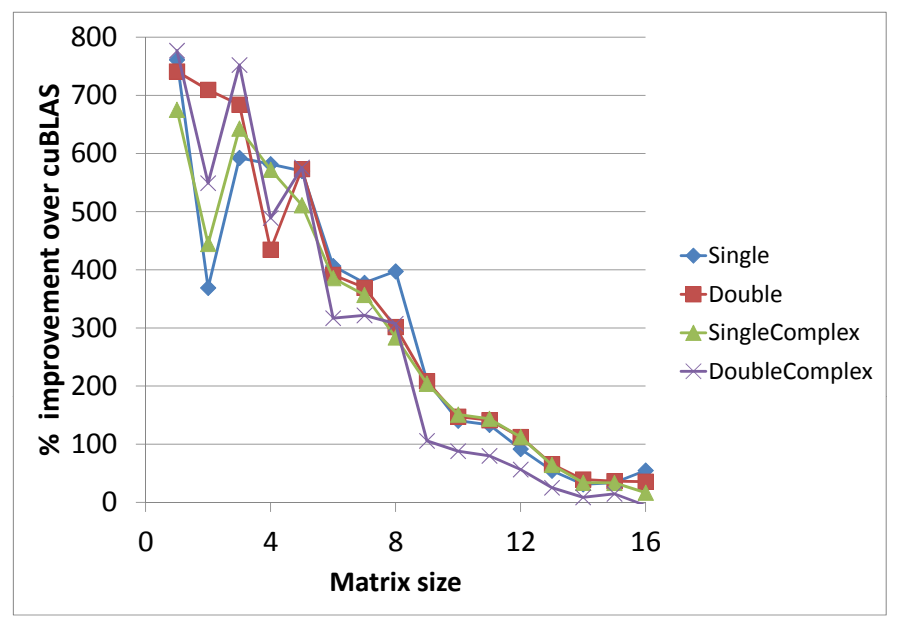

Figure 9: Relative improvement over cuBLAS batched implementation for various matrix sizes when our improved interface and implementation is used. 
research, we believe that a special batched GEMM implementation for this case will be much faster than using non-batched GEMM. Naturally, it will be faster than our case where both matrices vary.

The second leading dimension based interface presented here can be extended to other BLAS routines naturally, for GPUs or multi-core CPUs. As of now, we don't have data to quantify the importance of a batched GEMM implementation on GPU in high performance of other GPU-based BLAS routines for small matrices. However, just like the CPU case, we believe it will be crucial to formulate other batched BLAS kernels in terms of batched GEMM.

\section{Acknowledgements}

This work was partially supported by the US Department of Energy SBIR Grant DE-SC0004439. We thank the referees for a thorough reading and suggesting changes that led to improvements to the manuscript.

\section{References}

[1] R. Nath, S. Tomov, J. Dongarra, An Improved Magma Gemm For Fermi Graphics Processing Units, Int. J. High Perform. Comput. Appl. 24 (4) (2010) 511-515.

[2] K. Goto, R. Van De Geijn, High-performance implementation of the level-3 blas, ACM Trans. Math. Softw. 35 (1) (2008) 4:1-4:14.

[3] G. Karniadakis, S. Sherwin, Spectral/hp element methods for CFD, Oxford University Press, USA, 1999.

[4] M. Deville, P. Fischer, E. Mund, High-Order Methods for Incompressible Fluid Flow, Cambridge University Press, 2002.

[5] L. Demkowicz, Computing with hp-ADAPTIVE FINITE ELEMENTS: Volume I: One and Two Dimensional Elliptic and Maxwell Problems, Chapman \& Hall/CRC Press, 2006.

[6] L. Demkowicz, W. Rachowicz, D. Pardo, M. Paszynski, J. Kurtz, A. Zdunek, Computing with hp-ADAPTIVE FINITE ELEMENTS: Volume II Frontiers: Three Dimensional Elliptic and Maxwell Problems with Applications, Chapman \& Hall/CRC Press, 2007. 
[7] P. Šolín, K. Segeth, I. Doležel, Higher-order Finite Element Methods, Chapman \& Hall/CRC, 2003.

[8] P. Bientinesi, V. Eijkhout, K. Kim, J. Kurtz, R. van de Geijn, Sparse direct factorizations through unassembled hyper-matrices, Computer Methods in Applied Mechanics and Engineering 199 (2010) 430-438.

[9] B. Kågström, P. Ling, C. V. Loan, GEMM-based level 3 BLAS: highperformance model implementations and performance evaluation benchmark, ACM Transactions on Mathematical Software.

[10] NVIDIA CUDA Basic Linear Algebra Subroutines (cuBLAS) library, https://developer.nvidia.com/cublas, accessed: Feb 21, 2013.

[11] E. Anderson, Z. Bai, C. Bischof, S. Blackford, J. Demmel, J. Dongarra, J. Du Croz, A. Greenbaum, S. Hammarling, A. McKenney, D. Sorensen, LAPACK Users' Guide, 3rd Edition, SIAM, Philadelphia, PA, 1999.

[12] B. Stroustrup, The C++ Programming Language, 3rd Edition, AddisonWesley Longman Publishing Co., Inc., Boston, MA, USA, 2000.

[13] N. J. Higham, Stability of a method for multiplying complex matrices with three real matrix multiplications, SIAM J. Matrix Anal. Appl. 13 (3) (1992) 681-687. 


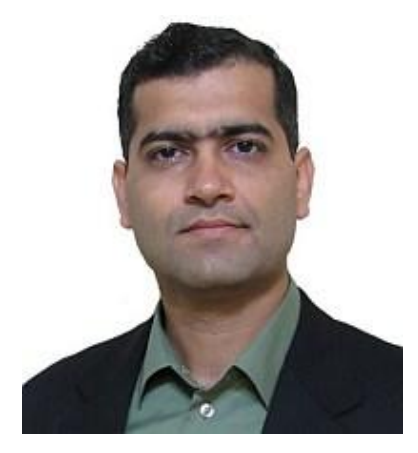

Dr. Chetan Jhurani received his Ph.D. in Computational and Applied Mathematics from the University of Texas at Austin in 2009. He worked with Prof. Leszek Demkowicz on homogenization of polymer elasticity problems. He is now a research mathematician at Tech- $X$ Corporation and works on designing algorithms and software for solving partial differential equations on CPUs and GPUs. His research interests include numerical homogenization, sparse and dense matrix algorithms, preconditioner design, numerical optimization, and automated motion planning.

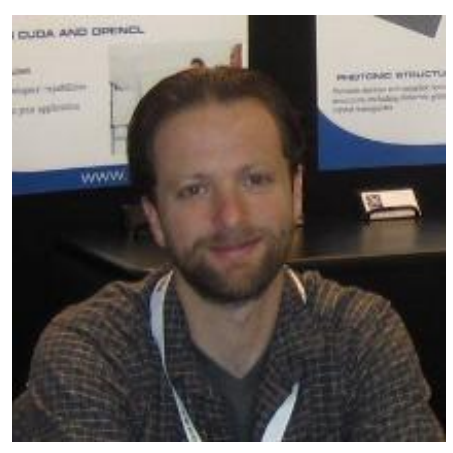

Dr. Paul Mullowney received his Ph.D. in applied mathematics from the University of Colorado at Boulder in December of 2004 under the guidance of Professors Keith Julien and James Meiss. His thesis work focused on particle kinetics/dynamics in 3D hydrodynamic convection problems. Upon completion of his Ph.D., Dr. Mullowney then went on to become a post doctoral fellow at the University of Canterbury in Christchurch, NZ. There, he worked on problems in mathematical biology which required modeling, simulation, and parameter estimation from experimentally gathered data sets. Since joining Tech-X Corporation in September of 2006, Dr. Mullowney has worked on many problems in GPU computing. In particular he has many new features to the PETSC open source library including GPU-accelerated kernels for various preconditioner algorithms and sparse matrix vector multiplication. 